\title{
Comportamento da função pulmonar, força muscular respiratória e qualidade de vida em pacientes submetidos às toracotomias eletivas
}

\author{
Pulmonary function, respiratory muscle strength and quality of life in patients \\ submitted to elective thoracotomies
}

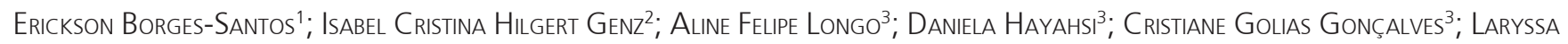
Milenkovich Bellinetti"; JoÃo Carlos Thomson ${ }^{5}$

R E S U M O

\begin{abstract}
Objetivo: Avaliar o comportamento da função pulmonar, força muscular respiratória e qualidade de vida no pré e pós-operatório de pacientes submetidos às toracotomias eletivas. Métodos: Foram avaliados 19 pacientes submetidos à toracotomia eletiva para obtenção dos parâmetros: capacidade vital forçada (CVF), volume expiratório forçado no primeiro segundo (VEF $)_{1}$, pressão inspiratória máxima (PImax), pressão expiratória máxima (PEmax) e qualidade de vida mediante aplicação do questionário SF-36. Os exames foram realizados no pré-operatório, $2^{\circ}, 10^{\circ}, 15^{\circ}, 30^{\circ}$ e $60^{\circ}$ dia de pós-operatório. Foram feitas análises de normalidade dos dados utilizando-se o teste de Shapiro-Wilk, análise descritiva das variáveis de estudo, bem como, análise de variância com comparações múltiplas utilizando-se os testes ANOVA e Friedman, com valor de $p<0,05$. Resultados: Houve significativa redução nas variáveis espirométricas e nas pressões respiratórias máximas no $2^{\circ}$ pós-operatório. CVF retornou aos valores pré-operatórios entre $015^{\circ} \mathrm{e}$ o $30^{\circ}$ pós-operatório, enquanto que $\mathrm{VEF}_{1}$ retornou entre o $10^{\circ}$ e $15^{\circ}$. PImax e PEmax retornam aos valores pré-operatórios entre o $10^{\circ}$ e $15^{\circ}$ pós-operatório. Houve redução da qualidade de vida nos domínios capacidade funcional e aspectos físicos, que retornaram aos valores pré-operatórios em até dois meses após o procedimento cirúrgico. Conclusão: Foi verificada significativa redução na função pulmonar e na força muscular respiratória, que retornaram aos valores basais em até 30 dias após o procedimento cirúrgico. Houve queda na qualidade de vida, que persistiu por até 60 dias após a operação.
\end{abstract}

Descritores: Cirurgia torácica. Testes de função respiratória. Qualidade de vida. Toracotomia. Capacidade vital.

\section{INTRODUÇÃO}

A incidência de complicações respiratórias pós-operatórias após as toracotomias varia de $10 \%$ a $40 \%{ }^{1}$. Elas, além de contribuírem significativamente para a morbidade e mortalidade peri-operatória, aumentam o tempo de internação e elevam os custos hospitalares ${ }^{2,3}$. Muitas se desenvolvem como resultado de mudanças nos volumes pulmonares que ocorrem, em parte, em resposta à disfunção dos músculos respiratórios ${ }^{3}$. Atribui-se à disfunção diafragmática pós-operatória redução entre $60 \%$ e $71 \%$ na capacidade vital, retornando este parâmetro aos valores pré-operatórios entre sete e 10 dias após a operação ${ }^{4}$.

Embora com indicação controversa, muita importância tem sido dada à avaliação da função pulmonar no pré e pós-operatório das laparotomias supraumbilicais e das toracotomias eletivas, especialmente em pacientes com sintomas respiratórios e nas ressecções pulmonares ${ }^{5,6}$.
A força muscular respiratória vem sendo estudada como fator preditivo para complicações nas toracotomias. Foi observado que ela, abaixo dos valores previstos no préoperatório, confere risco relativo maior para ocorrência de complicações nas toracotomias e laparotomias supraumbilicais ${ }^{7}$, bem como, foi sugerida a inclusão desta variável em escala de risco cirúrgico para pacientes submetidos à cirurgia cardíaca ${ }^{8}$. Os pacientes que não conseguem elevar seus valores de pressões respiratórias máximas no pós-operatório têm maior risco de desenvolverem complicações respiratórias 9 .

Nas toracotomias, principalmente nas ressecções pulmonares, há impacto negativo decorrente do procedimento cirúrgico na percepção da qualidade de vida que pode ser influenciado por diversos fatores físicos e emocionais, sendo a dor pós-operatória um importante fator limitante ${ }^{10}$.

Sugere-se que a intensidade da disfunção dos músculos respiratórios, com consequente diminuição da

Trabalho realizado no Hospital Universitário da Universidade Estadual de Londrina (HU-UEL) - Londrina (PR), Brasil.

1. Especialista em Fisioterapia Cardiorrespiratória pelo Instituto do Coração do Hospital das Clínicas da Faculdade de Medicina da Universidade de São Paulo (InCor - HCFMUSP)-SP-BR; 2. Aluna do curso de Residência em Fisioterapia Pulmonar pela Universidade Estadual de Londrina-PR-BR; 3. Residente em Fisioterapia Pulmonar pela Universidade Estadual de Londrina-PR-BR; 4. Docente do Departamento de Fisioterapia da Universidade Estadual de Londrina-PR-BR; 5. Docente do Departamento de Cirurgia Torácica da Universidade Estadual de Londrina-PR-BR. 
função pulmonar, posteriormente ao ato cirúrgico, concorra para aumento no período de restabelecimento da plena função respiratória, promovendo piora no estado funcional, favorecendo o desenvolvimento de complicações respiratórias e aumentando o tempo de retorno às atividades normais.

Sendo assim, o objetivo deste trabalho foi avaliar o comportamento da função pulmonar, da força muscular respiratória e da qualidade de vida no pré e pós-operatório de pacientes submetidos à toracotomias eletivas.

\section{MÉTODOS}

Foi realizado um estudo descritivo prospectivo do tipo série de casos. A coleta de dados foi realizada entre março de 2006 e abril de 2008. A amostra foi constituída por 19 pacientes provenientes do Setor de Cirurgia Torácica do Hospital Universitário da Universidade Estadual de Londrina. Todos os pacientes incluídos assinaram o Termo de Consentimento Livre e Esclarecido e a realização deste estudo foi aprovada pelo Comitê de Ética em Pesquisa da Universidade Estadual de Londrina sob parecer de número 161/05.

Foram incluídos pacientes com idade acima de 13 anos, internados para a realização de cirurgia torácica não-cardíaca eletiva a céu aberto, sob anestesia geral.

Os critérios de exclusão foram: 1) pacientes não colaborativos; 2) em uso de psicotrópicos, imunossupressores nos últimos 30 dias ou relaxantes musculares nas 24 horas precedentes ao exame; 3) com presença de infecção pulmonar ocorrida entre o momento da avaliação pré-operatória e a operação. Também seriam excluídos os pacientes que se submeteram à reintervenção cirúrgica durante o período de acompanhamento do estudo, bem como, aqueles que deixaram de ser acompanhados pelos avaliadores desta pesquisa em mais de dois momentos do pós-operatório.

No pré-operatório foram obtidos os seguintes dados: idade, sexo, tabagismo (foi considerado fumante o paciente que referiu ter fumado no mínimo um cigarro por dia por mais de um ano até então, ou mais de 20 maços durante toda a vida), presença de sintomas respiratórios (tosse, expectoração ou dispnéia), doença pulmonar prévia (doença obstrutiva ou restritiva crônica diagnosticada previamente ou durante o período de internação) comorbidades (hipertensão arterial, diabete melito e/ou cardiopatia), índice de massa corpórea, espirometria, força muscular respiratória e qualidade de vida.

No transoperatório foi pesquisado o tempo de operação (maior ou menor que 210 minutos) e o tempo de anestesia.

Foi feito o acompanhamento do paciente no $2^{\circ}$ $10^{\circ}, 15^{\circ}, 30^{\circ}$ e $60^{\circ}$ dia de pós-operatório, no qual foram avaliadas a força muscular respiratória, função pulmonar, qualidade de vida e ocasional ocorrência de complicações respiratórias (pneumonia, atelectasia com repercussão clínica evidente, broncoespasmo, ventilação mecânica prolongada por mais que 48 horas, derrame pleural ou pneumotórax, insuficiência respiratória aguda ou óbito).

Realizou-se a espirometria para obtenção dos parâmetros: Capacidade Vital Forçada (CVF) e Volume Expiratório Forçado no Primeiro Segundo $\left(\mathrm{VEF}_{1}\right)$ de acordo com as recomendações da II Diretriz Brasileira de Testes de Função Pulmonar ${ }^{11}$. A técnica foi explicada e demonstrada ao paciente, devendo ele realizar os testes em posição sentada, em uso de prendedor nasal e com o bocal firmemente acoplado entre os dentes e lábios. Foram executados no mínimo três testes válidos, sendo registrado o maior valor obtido, desde que não fosse o último avaliado. Utilizaram-se aparelhos das marcas Pony (Cosmed, Itália) e Respiradyne (Sherwood, USA), sendo que cada paciente foi avaliado em todo o seguimento do estudo por um único aparelho.

A força muscular respiratória foi medida pelas pressões respiratórias máximas: Pressão Inspiratória Máxima (PImax) e Pressão Expiratória Máxima (PEmax) ${ }^{12}$, utilizando-se um manovacuômetro analógico (Makil - Londrina, PR, Brasil), com escala de -200 a $200 \mathrm{~cm}$ de $\mathrm{H}_{2} \mathrm{O}$. O paciente permanecia sentado, com prendedor nasal e utilizando bocal plástico rígido. A técnica foi repetida até que fossem obtidos os valores de três manobras válidas, com variação menor que 10\% entre elas e com intervalos de um minuto para descanso. Foi registrado o maior valor sustentado obtido, desde que não fosse o último medido.

Os testes de função pulmonar e de força muscular respiratória foram realizados no período pré-operatório e no $2^{\circ}, 10^{\circ}, 15^{\circ}, 30^{\circ}$ e $60^{\circ}$ dia do pós-operatório. Os valores de CVF, VEF ${ }_{1}$, PImax e PEmax foram comparados de acordo com as médias e porcentagens obtidas, dividindose os valores obtidos no pós-operatório pelos valores préoperatórios e multiplicando-os por 100.

A Qualidade de Vida foi avaliada mediante aplicação do questionário genérico de qualidade de vida Medical Outcomes Study 36 - Item Short-Form Health Survey (SF-36) ${ }^{13}$, validado para a língua portuguesa. Este questionário incluiu a investigação de oito domínios: capacidade funcional, aspectos físicos, dor, estado geral de saúde, vitalidade, aspectos sociais, aspectos emocionais e saúde mental. Embora sendo questionário autoaplicável, optouse por ser aplicado pelo avaliador. A aplicação do questionário se deu no período pré-operatório, $30^{\circ}$ e $60^{\circ}$ do pósoperatório.

Os pacientes foram operados por uma mesma equipe, com técnicas cirúrgica e anestésica semelhantes. Todos foram acompanhados pelo Serviço de Fisioterapia com esquemas semelhantes de atendimento nos períodos pré e pós-operatório. As avaliações desta pesquisa foram realizadas por uma única equipe, que previamente recebeu treinamento semelhante.

Foram feitas análises de normalidade dos dados utilizando-se o teste de Shapiro-Wilk, análise descritiva das 
variáveis de estudo pelo cálculo das médias, desvios-padrão, medianas e intervalos interquartílicos. Realizou-se análise de variância com comparações múltiplas utilizando-se os testes ANOVA e Friedman com os pós-testes de Tukey e Dunns. O nível de significância adotado foi de 0,05 para o erro á.

\section{RESULTADOS}

Os dados antropométricos e funcionais dos 19 indivíduos avaliados estão descritos em média e desviopadrão na tabela 1.

Quanto às variáveis de função pulmonar e força muscular respiratória, observou-se importante redução dos parâmetros espirométricos e das pressões respiratórias máximas no pós-operatório imediato; apresentando estes incrementos progressivos ao longo do período de estudo. A análise evolutiva das médias e desvios-padrão de CVF, $\mathrm{VEF}_{1}$, PImax e PEmax está apresentada na tabela 2.

Foi encontrada queda da CVF média de $41,5 \%$, $30,7 \%, 25,08 \%, 20,1 \%$ e $15,5 \%$ no $2^{\circ}, 10^{\circ}, 15^{\circ}, 30^{\circ}$ e $60^{\circ}$ dia do pós-operatório em relação aos valores de préoperatório. Houve restituição de CVF entre o $15^{\circ}$ e $30^{\circ}$ dias $(p>0,05)$. Quanto ao $\mathrm{VEF}_{1}$ observou-se o mesmo

Tabela 1 - Caracterização da população de estudo com valores absolutos e porcentagens ou médias e desvios-padrão.

\begin{tabular}{lcc}
\hline \multicolumn{1}{c}{ Variáveis } & População & de Estudo \\
\hline Gênero: $\quad$ Masculino & 10 & $(52,6 \%)$ \\
& 9 & $(47,4 \%)$ \\
Idade (anos)* & 43 & \pm \\
IMC $\left(\mathrm{kg} / \mathrm{m}^{2}\right)^{*}$ & $21,5 \pm$ & 4 \\
Tabagistas & 10 & $(52,6 \%)$ \\
Portadores de pneumopatia prévia & 9 & $(47,4 \%)$ \\
Portadores de sintomas respiratórios & 12 & $(63,2 \%)$ \\
Portadores de comorbidades & 6 & $(31,2 \%)$ \\
Tempo de operação (minutos)* & $198,3 \pm 130$ \\
Tempo de anestesia (minutos)* & $269,6 \pm 140$ \\
Toracotomia sem ressecção & 10 & $(52,6 \%)$ \\
\hline
\end{tabular}

* Valores expressos em média \pm desvio-padrão

IMC: índice de massa corpórea. comportamento apresentado por CVF, com redução de $48,7 \%, 26,1 \%, 22,2 \% 14,1 \%$ e $11,1 \%$ dos valores apresentados no pré-operatório. O VEF 1 equiparou-se aos valores basais entre o $10^{\circ}$ e o $15^{\circ}$ dia do pós-operatório $(p>0,05)$ (Figura 1).

A análise da força muscular respiratória, evidenciou importante queda dos valores de PImax ao longo do pós-operatório de $55 \%, 33,5 \%, 13 \%$ e $5,6 \%$ no $2^{\circ}, 10^{\circ}$, $15^{\circ}$ e $30^{\circ}$ dias, respectivamente. Para os valores de PEmax a redução foi de $58,1 \%, 31,4 \%, 14,4 \%$ e $2,3 \%$ no $2^{\circ}$, $10^{\circ}, 15^{\circ}$ e $30^{\circ}$ dia. No $60^{\circ}$ dia, os pacientes superaram seus valores pré-operatórios de PImax e PEmax (106,3\% e $106,6 \%$, respectivamente). Foi observada restituição tanto de PImax como de PEmax entre o $10^{\circ}$ e $15^{\circ}$ dias $(p>0,05)$ (Figura 2).

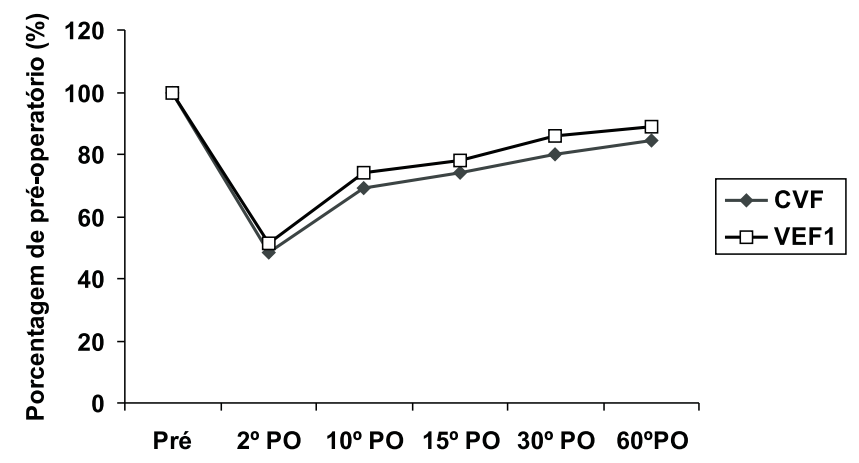

Figura 1 - Valores em percentual de pré-operatório de CVF e $V E F_{1}$.

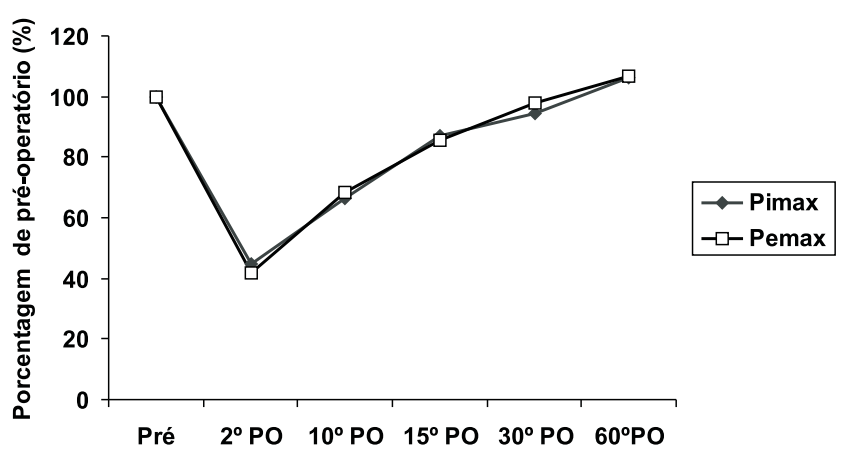

Figura 2 - Valores em percentual de pré-operatório de PImax e PEmax.

Tabela 2 - Médias e desvios-padrão de CVF, VEF, PImax e PEmax.

\begin{tabular}{|c|c|c|c|c|c|c|}
\hline Variável & Pré & $2^{\circ} \mathrm{PO}$ & $10^{\circ} \mathrm{PO}$ & $15^{\circ} \mathrm{PO}$ & $30^{\circ} \mathrm{PO}$ & $60^{\circ} \mathrm{PO}$ \\
\hline CVF (I) & $3,03 \pm \quad 0,86$ & $1,47 \pm 0,46$ & $2,1 \pm 0,7$ & $2,25 \pm 0,83$ & $2,42 \pm 0,81 *$ & $2,56 \pm 0,68$ \\
\hline VEF1 (I) & $2,34 \pm \quad 0,77$ & $1,20 \pm 0,33$ & $1,73 \pm 0,57$ & $1,82 \pm 0,68 *$ & $2,01 \pm 0,62$ & $2,08 \pm 0,54$ \\
\hline $\operatorname{PImax}\left(\mathrm{cmH}_{2} \mathrm{O}\right)$ & $84,1 \pm 32,4$ & $37,9 \pm 19,7$ & $55,9 \pm 29,7$ & $73,2 \pm 35,1^{*}$ & $79,4 \pm 42,3$ & $89,4 \pm 39,7$ \\
\hline $\operatorname{PEmax}\left(\mathrm{cmH}_{2} \mathrm{O}\right)$ & $98,2 \pm 34,1$ & $41,2 \pm 16,9$ & $67,4 \pm 22,2$ & $84,1 \pm 31,1$ * & $95,9 \pm 34,8$ & $104,7 \pm 31,6$ \\
\hline
\end{tabular}

* $p>0,05$ 
De acordo com a tabela 3, ao se avaliar a qualidade de vida, observou-se redução clinicamente significante em todos os parâmetros avaliados. Entretanto, essa redução não foi considerada estatisticamente significante para a maioria dos domínios. No domínio "capacidade funcional" foi encontrada diferença estatisticamente significante entre $030^{\circ}$ e $60^{\circ}$ dias do pós-operatório $(p=0,02)$ e no domínio "aspectos físicos" houve diferença estatisticamente significante entre o pré e o $30^{\circ}$ dia $(p=0,0005)$.

O tempo médio de internação hospitalar pósoperatório foi $11 \pm 9$ dias, sendo que dos 19 pacientes avaliados, 12 necessitaram de assistência em UTI por pelo menos um dia. Apenas um paciente evoluiu com complicação respiratória, sendo diagnosticada como pneumonia.

\section{DISCUSSÃO}

Este estudo avaliou o comportamento da função pulmonar, da força muscular respiratória e da qualidade de vida em pacientes submetidos à toracotomias eletivas. Os resultados mostraram que houve significativa redução nos volumes e capacidades pulmonares, bem como, na força muscular respiratória no período pós-operatório.

A redução na função pulmonar que ocorre no pós-operatório das toracotomias está associada à mudanças no padrão ventilatório, retenção de $\mathrm{CO}_{2}$, redução da pressão arterial de $\mathrm{O}_{2}$ e prejuízo nos mecanismos de defesa pulmonar ${ }^{2,14}$, podendo evoluir para complicações respiratórias quando ocorre desvio do resultado esperado para o procedimento cirúrgico ${ }^{15}$.

Neste estudo, no $2^{\circ}$ dia do pós-operatório, houve queda de $41,5 \%$ nos valores de CVF e de $48,7 \%$ no $V_{E F}$, sendo esses resultados convergentes com os de outros autores ${ }^{16,17}$. Na análise evolutiva dos parâmetros espirométricos, CVF retornou aos valores pré-operatórios entre o $15^{\circ}$ e o $30^{\circ}$ dia, enquanto que o VEF, retornou entre $010^{\circ}$ e $15^{\circ}$. Esses resultados foram semelhantes aos de outros autores que estudaram as mudanças na função respiratória no pós-operatório de operações torácicas e abdominais superiores ${ }^{18,19}$.
Na amostra estudada, houve queda de 55\% para os valores de PImax e 58,1\% para os valores de PEmax no $2^{\circ}$ dia do pós-operatório, tendo retornado aos valores préoperatórios entre o $10^{\circ}$ e $15^{\circ}$. Estes resultados foram semelhantes aos da literatura consultada ${ }^{9}$. Estudos sobre operações de risco, realizados durante o período de internação hospitalar ou com tempo de seguimento inferior a oito dias de pós-operatório, não foram capazes de detectar o retorno da função pulmonar e da força muscular respiratóriaa 20,21 .

A redução dos volumes pulmonares vem acompanhada de diminuição da complacência pulmonar, com aumento do trabalho elástico do pulmão. Essas alterações da mecânica respiratória exigem dos músculos respiratórios maior geração de pressão para que ocorra a expansibilidade pulmonar. Quando esse mecanismo de ação da bomba ventilatória está ineficiente ocorre a instalação de áreas de atelectasia com consequente retenção de secreção ${ }^{14}$, sendo que normalização mais precoce da função respiratória nas operações de risco estaria relacionada à menor incidência de complicações respiratórias ${ }^{19}$.

Todos os pacientes foram submetidos à fisioterapia respiratória no pré e pós-operatórios. Tanto no pré como no pós-operatório eles receberam orientações sobre a operação, sobre a importância da deambulação precoce e da tosse, realizaram exercícios de cinesioterapia respiratória e foram submetidos à técnicas de re-expansão pulmonar, sem protocolo específico, conforme necessidades individuais.

A literatura afirma que a realização de fisioterapia é mais eficaz em prevenir complicações do que a sua não realização ${ }^{20,22}$. Estudos sugerem os benefícios da realização de técnicas de re-expansão pulmonar ${ }^{23}$, cinesioterapia respiratória ${ }^{19}$, estímulo à tosse e deambulação precoce ${ }^{22}$ em pacientes submetidos à operação de risco.

O treinamento muscular respiratório tem sido extensamente pesquisado e os resultados obtidos apontam êxito na utilização desta conduta fisioterapêutica na prevenção de complicações respiratórias. Diversos autores constataram que o treinamento muscular respiratório pré e pós-operatório melhora a função pulmonar, a força mus-

Tabela 3 - Comportamento da qualidade de vida.

\begin{tabular}{lccc}
\hline Domínios SF-36 & Pré-operatório & $3^{\circ}$ PO & $60^{\circ}$ PO \\
\hline Capacidade funcional & $68,6 \pm 28,9$ & $61,8 \pm 23,6$ & $76,4 \pm 18,9 *$ \\
Aspectos físicos & $56,9 \pm 41,8$ & $13,9 \pm 17,6^{*}$ & $36,1 \pm 38,6$ \\
Dor & $64,4 \pm 36,9$ & $56,1 \pm 27,7$ & $63,6 \pm 26,4$ \\
Estado geral de saúde & $74,8 \pm 20,7$ & $68 \pm 23,2$ & $71,4 \pm 22,4$ \\
Vitalidade & $72,2 \pm 25,6$ & $61,6 \pm 27,9$ & $65,8 \pm 24,5$ \\
Aspectos sociais & $55,6 \pm 33$ & $61,6 \pm 37,8$ & $68,8 \pm 27,5$ \\
Aspectos emocionais & $51,8 \pm 43,1$ & $38,4 \pm 37,6$ & $53,7 \pm 39,8$ \\
Saúde mental & $67,6 \pm 25,7$ & $68 \pm 30,6$ & $66,8 \pm 25,4$
\end{tabular}

* $p<0,05$ 
cular respiratória ${ }^{9,24}$, reduz a incidência de complicações respiratórias ${ }^{25}$ e diminui o tempo de internação hospita$\operatorname{lar}^{9,26}$. Entretanto, para que esta técnica seja adequadamente empregada é necessário o monitoramento da força muscular respiratória que o paciente apresenta para tornar precisa a carga a ser imposta ${ }^{27}$, o que certamente justifica a sua pontual avaliação.

Neste estudo, houve piora da qualidade de vida no parâmetro "aspectos físicos". Outros autores ${ }^{10,28,29}$ também atribuem essa piora, principalmente, aos domínios "capacidade funcional" e "aspectos físicos", relacionando-a à pior função pulmonar, pior capacidade aeróbia e, nos casos de neoplasia, pior prognóstico.

A incidência de complicações nas toracotomias varia de $10 \%$ a $40 \%{ }^{1}$. Neste estudo apenas um paciente
(5,26\%) apresentou CRP, diagnosticada como pneumonia. A realização de fisioterapia, aliada ao maior critério por parte da equipe envolvida na avaliação da operabilidade dos pacientes, parece ter influenciado na baixa incidência de complicações ou óbito nesta pesquisa.

Conclui-se que houve significativa redução na função pulmonar e na força muscular respiratória no pósoperatório das toracotomias eletivas. Foi constatada normalização da CVF entre o $10^{\circ}$ e o $15^{\circ}$ dias do pós-operatório e de $\mathrm{VEF}_{1}$ entre o $10^{\circ}$ e o $15^{\circ}$ dia. Tanto PImax como PEmax normalizaram-se entre $010^{\circ}$ e o $15^{\circ}$ dia. Houve piora da qualidade de vida nos domínios capacidade funcional e aspectos físicos, detectados um mês após a operação, retornando aos valores pré-operatórios em até dois meses após o procedimento cirúrgico.

\title{
A B S T R A C T
}

\begin{abstract}
Objective: To evaluate pre-and postoperative pulmonary function, respiratory muscle strength and quality of life in patients submitted to elective thoracotomy. Methods: We selected 19 patients undergoing elective thoracotomy to assess the following parameters: forced vital capacity (FVC), forced expiratory volume in one second (FEV1), maximal inspiratory pressure (MIP), maximal expiratory pressure (MEP) and quality of life through implementation of the SF-36. The examinations were performed preoperatively and in the second, 10th, 15th, 30th and 60th days postoperatively. We conduced data normality analyzes were made with the Shapiro-Wilk test, descriptive analysis of the study variables, as well as analysis of variance with multiple comparisons using ANOVA and Friedman, $p$-value $<0.05$. Results: There was a significant decrease in spirometric levels and in maximal respiratory pressures in the second postoperative day. FVC returned to preoperative values between the 15th and 30th postoperative days, whereas FEV1, between the 10th and 15th. MIP and MEP returned to preoperative values between the 10th and 15th postoperative days. There was a decrease in quality of life as for functional capacity and physical aspects, which returned to preoperative values within two months after surgery. Conclusion: There was significant reduction in lung function and respiratory muscle strength, which returned to baseline within 30 days after surgery. There was a decrease in quality of life that persisted for up to 60 days after the operation.
\end{abstract}

Key words: Thoracic surgery. Respiratory function tests. Quality of life. Thoracotomy. Vital capacity.

\section{REFERENCIAS}

1. Arozullah AM, Conde MV, Lawrence VA. Preoperative evaluation for postoperative pulmonary complications. Med Clin North Am. 2003;87(1):153-73

2. Smetana GW. Preoperative pulmonary evaluation. N Engl J Med. 1999;340(12):937-44

3. Ferguson MK. Preoperative assessment of pulmonary risk. Chest. 1999;115(5 Suppl):58S-63S.

4. Tisi GM. Preoperative evaluation of pulmonary function. Am Rev Respir Dis. 1979;119(2):293-310.

5. Pereira ED, Fernandes AL, da Silva Anção M, de Araúja Pereres $C$, Atallah AN, Faresin SM. Prospective assessment of the risk of postoperative pulmonary complications in patients submitted to upper abdominal surgery. Sao Paulo Med J. 1999;117(4):151-60.

6. Saad IA, Zambon L. Clinical variables of preoperative risk. Rev Assoc Med Bras. 2001;47(2):117-24.

7. Bellinetti LM, Thomson JC. Respiratory muscle evaluation in elective thoracotomies and laparotomies of the upper abdomen. J Bras Pneumol. 2006;32(2):99-105.

8. Hulzebos EH, Van Meeteren NL, De Bie RA, Dagnelie PC, Helders PJ. Prediction of postoperative pulmonary complications on the basis of preoperative risk factors in patients who had undergone coronary artery bypass graft surgery. Phys Ther. 2003;83(1):8-16.
9. Nomori H, Kobayashi R, Fuyuno G, Morinaga S, Yashima H. Preoperative respiratory muscle training. Assessment in thoracic surgery patients with special reference to postoperative pulmonary complications. Chest. 1994;105(6):1782-8.

10. Saad IA, Botega NJ, Toro IF. Evaluation of quality of life of patients submitted to pulmonary resection due to neoplasia. J Bras Pneumol. 2006;32(1):10-5

11. Sociedade Brasileira de Pneumologia e Tisiologia. Diretrizes para testes de função pulmonar 2002. J Bras Pneumol. 2002;28(Supl 3).

12. American Thoracic Society/European Respiratory Society. ATS/ ERS Statement on respiratory muscle testing. Am J Respir Crit Care Med. 2002;166(4):518-624.

13. Ciconelli RM, Ferraz MB, Santos W, Meinão I, Quaresma MR. Tradução para a língua portuguesa e validação do questionário genérico de avaliação de qualidade de vida SF-36 (Brasil SF-36). Rev Bras Reumatol. 1999;39(3):143-50.

14. Siafakas NM, Mistrouska I, Bouros D, Georgopoulos D. Surgery and the respiratory muscles. Thorax. 1999;54(5):458-65.

15. Pezzella AT, Adebonojo SA, Hooker SG, Mabogunje OA, Colan AA. Complications of general thoracic surgery. Curr Probl Surg. 2000;37(11):733-858.

16. Giacomazzi CM, Lagni VB, Monteiro MB. A dor pós-operatória como contribuinte do prejuízo na função pulmonar em pacientes submetidos à cirurgia cardíaca. Braz J Cardiovasc Surg. 2006;21(4):386-92. 
17. Varela G. Brunelli A, Rocco G, Marasco R, Jiménez MF, Sciarra V, et al. Predicted versus observed FEV1 in the immediate postoperative period after pulmonary lobectomy. Eur Cardiothorac Surg. 2006;30(4):644-8.

18. Pecora DV. Predictability of effect of abdominal and thoracic surgery upon pulmonary function. Ann Surg. 1969;170(1):101-8.

19. Gastaldi AC, Magalhães CMB, Baraúna MA, Silva EMC, Souza HCD. Benefícios da cinesioterapia respiratória no pós-operatório de colecistectomia laparoscópica. Rev Bras Fisioter. 2008;12(2):1006.

20. Paisani DM, Chiavegato LD, Faresin SM. Volumes, capacidades pulmonares e força muscular respiratória no pós-operatório de gastroplastia. J bras pneumol. 2005;31(2):125-32.

21. Lima VP, Bonfim D, Risso TT, Paisani Dde M, Fiore JF Jr, Chiavegato $L D$, et al. Influence of pleural drainage on postoperative pain, vital capacity and six-minute walk test after pulmonary resection. J Bras Pneumol. 2008;34(12):1003-7.

22. Faresin SM. Avaliação pré-operatória pulmonar. Rio de Janeiro: Revinter; 2005.

23. Lawrence VA, Cornell JE, Smetana GW; American College of Physicians. Strategies to reduce postoperative pulmonary complications after noncardiothoracic surgery: systematic review for the American College of Physicians. Ann Intern Med. 2006;144(8):596-608.

24. Weiner $P$, Man A, Weiner M, Rabner M, Waizman J, Magadle R, et al. The effect of incentive spirometry and inspiratory muscle training on pulmonary function after lung resection. J Thorac Cardiovasc Surg. 1997;113(3):552-7.

25. Dronkers J, Veldman A, Hoberg E, van der Waal C, van Meeteren N. Prevention of pulmonary complications after upper abdominal surgery by preoperative intensive inspiratory muscle training: a randomized controlled pilot study. Clin Rehabil. 2008;22(2):13442.
26. Hulzebos EH, Helders PJ, Favié NJ, De Bie RA, Brutel de la Riviere A, Van Meeteren NL. Preoperative intensive inspiratory muscle training to prevent postoperative pulmonary complications in highrisk patients undergoing $C A B G$ surgery: a randomized clinical trial JAMA. 2006;296(15):1851-7.

27. Elias DG, Costa D, Oishi J, Pires VA, Silva MAM. Efeitos do treinamento muscular respiratório no pré e pós-operatório de cirurgia cardíaca. Rev bras ter intensa. 2000;12(1):9-18.

28. Franceschini J, Santos AA, El Mouallem I, Jamnik S, Uehara C, Fernandes $A L$, et al. Assessment of the qualitey of life of patients with lung cancer using the Medical Outcomes Study 36-item Short-Form Health Survey. J Bras Pneumol. 2008;34(6):387-93.

29. Hamacher J, Büchi S, Gerogescu CL, Stammberger U, Thurnheer $\mathrm{R}$, Bloch $\mathrm{KE}$, et al. Improved quality of life after lung volume reduction surgery. Eur Respir J. 2002;19(1):54-60.

Recebido em 12/03/2011

Aceito para publicação em 19/05/2011

Conflito de interesse: nenhum

Fonte de financiamento: Conselho Nacional de Desenvolvimento $\mathrm{Ci}$ entífico e Tecnológico (CNPq).

\section{Como citar este artigo:}

Borges-Santos E, Genz ICH, Longo AF, Hayahsi D, Gonçalves CG, Bellinetti LM, Thomson JC. Comportamento da função pulmonar, força muscular respiratória e qualidade de vida em pacientes submetidos às toracotomias eletivas. Rev Col Bras Cir. [periódico na Internet] 2012 39(1). Disponível em URL: http://www.scielo.br/rcbc

\section{Endereço para correspondência:}

Erickson Borges Santos

E-mail: erick_borges@hotmail.com 